\title{
Mendelian randomization does not support serum calcium in prostate cancer risk
}

\author{
James Yarmolinsky ${ }^{1,2} \cdot$ Katie Berryman $^{1,2} \cdot$ Ryan Langdon $^{1,2} \cdot$ Carolina Bonilla $a^{1,2,3}$ on behalf of PRACTICAL \\ consortium · George Davey Smith ${ }^{1,2,4} \cdot$ Richard M. Martin ${ }^{1,2,4} \cdot$ Sarah J. Lewis ${ }^{1,2}$
}

Received: 3 July 2018 / Accepted: 11 September 2018 / Published online: 10 October 2018

(c) The Author(s) 2018

\begin{abstract}
Purpose Observational studies suggest that dietary and serum calcium are risk factors for prostate cancer. However, such studies suffer from residual confounding (due to unmeasured or imprecisely measured confounders), undermining causal inference. Mendelian randomization uses randomly assigned (hence unconfounded and pre-disease onset) germline genetic variation to proxy for phenotypes and strengthen causal inference in observational studies. We tested the hypothesis that serum calcium is associated with an increased risk of overall and advanced prostate cancer.

Methods A genetic instrument was constructed using five single-nucleotide polymorphisms robustly associated with serum calcium in a genome-wide association study $(n \leq 61,079)$. This instrument was then used to test the effect of a $0.5 \mathrm{mg} / \mathrm{dL}$ increase (1 standard deviation, SD) in serum calcium on risk of prostate cancer in 72,729 men in the PRACTICAL (Prostate Cancer Association Group to Investigate Cancer Associated Alterations in the Genome) Consortium (44,825 cases, 27,904 controls) and risk of advanced prostate cancer in 33,498 men (6,263 cases, 27,235 controls).

Results We found weak evidence for a protective effect of serum calcium on prostate cancer risk (odds ratio [OR] per $0.5 \mathrm{mg} /$ dL increase in calcium: $0.83,95 \%$ CI $0.63-1.08 ; p=0.12$ ). We did not find strong evidence for an effect of serum calcium on advanced prostate cancer (OR per $0.5 \mathrm{mg} / \mathrm{dL}$ increase in calcium: $0.98,95 \%$ CI $0.57-1.70 ; p=0.93$ ).

Conclusions Our Mendelian randomization analysis does not support the hypothesis that serum calcium increases risk of overall or advanced prostate cancer.
\end{abstract}

Keywords Diet $\cdot$ Nutrition $\cdot$ Calcium $\cdot$ Prostate cancer $\cdot$ Mendelian randomization

Abbreviations
GWAS
HR

Members from the Prostate Cancer Association Group to Investigate Cancer Associated Alterations in the Genome (PRACTICAL) consortium are provided in the Supplement notes. Information of the consortium can be found at http://practical.icr. ac.uk/.

Electronic supplementary material The online version of this article (https://doi.org/10.1007/s10552-018-1081-5) contains supplementary material, which is available to authorized users.

Sarah J. Lewis

S.J.Lewis@bristol.ac.uk

1 MRC Integrative Epidemiology Unit, University of Bristol, Bristol, UK

2 Population Health Sciences, Bristol Medical School, University of Bristol, Bristol, UK
InSIDE Instrument strength independent of direct effect

IV Instrumental variable

MR Mendelian randomization

OR Odds ratio

PRACTICAL Prostate Cancer Association Group to Investigate Cancer Associated Alterations in the Genome

RR Risk ratio

SNPs Single-nucleotide polymorphisms

WME Weighted median estimator

3 Department of Preventive Medicine, School of Medicine, University of São Paulo, São Paulo, Brazil

4 National Institute for Health Research (NIHR) Bristol Biomedical Research Centre, University Hospitals Bristol NHS Foundation Trust and the University of Bristol, Bristol, UK 


\section{Introduction}

Prostate cancer is the most frequently diagnosed cancer among men globally and is a common cause of male cancer death [1]. Despite the considerable global burden attributed to prostate cancer, to date few risk factors (advanced age, ethnicity, family history of prostate cancer) have been identified and no modifiable risk factors have been established for this condition [2]. Nonetheless, global variation in prostate cancer mortality [3, 4] and findings from migration studies (i.e. the convergence toward local prostate cancer mortality rates among men who migrate from non-Western to Western populations) [5-7] provide support for a role of modifiable risk in prostate carcinogenesis.

Dietary calcium intake has been associated with an increased risk of prostate cancer in prospective epidemiological studies [8-10]. In a meta-analysis of fifteen prospective studies, high dietary calcium intake, as compared to low intake, was associated with an $18 \%$ (95\% CI 8-30\%) increased prostate cancer risk [11]. Similarly, high calcium intake has been linked to an increased risk of advanced [12-14] and fatal prostate cancer [13], though findings have been inconsistent $[15,16]$. Though serum calcium is normally tightly regulated in the body and does not fluctuate substantially across levels of dietary calcium intake [17, 18], Giovannucci proposed that higher dietary calcium may influence risk of prostate cancer by lowering circulating levels of $1,25(\mathrm{OH})_{2}$ vitamin $\mathrm{D}$, a presumed tumour suppressor, [19-21] in order to achieve calcium homeostasis [22]. A more direct method of testing the hypothesis that calcium metabolism influences prostate carcinogenesis would be to examine the association of serum calcium levels with prostate cancer risk. However, studies examining the association of pre-diagnostic serum calcium levels with incident or fatal prostate cancer [23, 24], or post-diagnostic serum calcium with prostate cancer survival $[25,26]$, have generated conflicting results: some report positive associations of serum calcium with prostate cancer [23, 26, 27], whereas others have been compatible with a null effect [23-25, 28, 29].

Establishing a causal role of elevated serum calcium in prostate carcinogenesis could have therapeutic implications for the prevention or treatment of prostate cancer. However, obtaining reliable estimates of causal effects from observational studies is a challenge as these studies are prone to various biases including residual confounding (due to unmeasured or imprecisely measured confounders) and exposure measurement error which can undermine robust causal inference [30, 31].

Mendelian randomization (MR) is an analytical approach that uses randomly assigned (hence unconfounded and pre-disease) germline genetic variants as instruments (i.e. proxies for the risk factor of interest) to examine the causal effects of risk factors on health outcomes [32, 33]. MR is a form of instrumental variable (IV) analysis that allows for unbiased causal effects to be estimated if three assumptions are met: (1) the instrument (e.g. a single germline genetic variant or a multi-allelic score) is robustly associated with the exposure of interest; (2) the instrument is not associated with any confounding factor(s) that would otherwise distort the association between the exposure and outcome; and (3) there is no pathway through which an instrument influences an outcome except through the exposure (known as the "exclusion restriction criterion"). The random allocation of genetic variants at conception and the independent assortment of parental alleles at meiosis means that, at a population level, analyses using genetic variants as instruments for a risk factor of interest should not be confounded by environmental and lifestyle factors that typically distort observational studies.

The availability of germline genetic variants (SNPssingle-nucleotide polymorphisms) robustly associated with serum calcium and prostate cancer in separate and independent genome-wide association studies (GWAS) [34, 35] can permit examination of the causal effect of increased serum calcium on prostate cancer risk using a "two-sample Mendelian randomization" framework [36]. Such an approach provides an efficient and statistically robust method of appraising causal relationships between traits, bypassing the need to have access to complete phenotypic and genotypic data on all participants in one sample.

Given uncertainty surrounding the role of serum calcium in prostate cancer aetiology and progression, we used data from: (i) a GWAS of serum calcium in up to 61,079 individuals of European descent; and (ii) a GWAS of prostate cancer in men of European descent $(n=72,729)$. These samples were used to perform a two-sample Mendelian randomization analysis to examine the causal effect of elevated serum calcium with risk of overall and advanced prostate cancer.

\section{Materials and methods}

\section{Prostate cancer population}

We obtained summary genome-wide association study (GWAS) statistics from analyses on 44,825 men with prostate cancer and 27,904 control men of European descent from 108 studies in the Prostate Cancer Association Group to Investigate Cancer Associated Alterations in the Genome (PRACTICAL) consortium [35]. Summary statistics were also obtained from analyses on 6,263 men with advanced prostate cancer (defined as Gleason score $\geq 8$, prostate-specific antigen $>100 \mathrm{ng} / \mathrm{mL}$, metastatic disease (M1), or death from prostate cancer) and 27,235 controls. All studies in PRACTICAL have the relevant Institutional Review Board 
approval from each country, in accordance with the Declaration of Helsinki. Genotype data were obtained by either direct genotyping using an Illumina Custom Infinium array (OncoArray) consisting of approximately 530,000 SNPs [37] or by imputation with reference to the 1000 Genomes Project Phase Three dataset [38]. All SNPs with a poor imputation quality $\left(r^{2}<0.30\right)$, a minor allele frequency of $<1 \%$, a call rate of $<98 \%$, or evidence of violation of Hardy-Weinberg equilibrium ( $p<10^{-7}$ in controls or $p<10^{-12}$ in cases) were removed. Analyses were performed across individual studies in PRACTICAL using logistic regression in models that were adjusted for the first seven principal components of ancestry (to control for population stratification) and study relevant covariates. Results were meta-analysed across the PRACTICAL studies using an inverse-variance fixed-effects approach to give an overall effect-estimate.

\section{Calcium-associated SNP selection}

SNPs to proxy for serum calcium were obtained from a GWAS meta-analysis of 39,400 individuals of European descent from 17 population-based cohorts [34]. Genetic instruments were constructed by obtaining SNPs shown to robustly $\left(p<1 \times 10^{-7}\right)$ and independently to associate $\left(r^{2}<0.01\right)$ with serum calcium levels that were replicated (one-sided $p<0.05$ ) in an independent meta-analysis of up to 21,679 individuals of European descent. In total, seven SNPs located in or near CASR (rs1801725), DGKD (rs1550532), GCKR (rs780094), GATA3 (rs10491003), CARS (rs7481584), DGKH (rs7336933), and CYP24Al (rs1570669) were independently replicated. Summary data on rs1801725 were not available in the PRACTICAL OncoArray analysis so we used a proxy SNP located in CASR (rs17251221) in high linkage disequilibrium with rs1801725 $\left(r^{2}=0.85\right)$, using the 1000 Genomes Project CEU database as a reference [39]. As an initial test for horizontal pleiotropy (a single locus influencing multiple phenotypes through independent biological pathways; a violation of the "exclusion restriction criterion"), we examined associations of calcium SNPs with thousands of other traits in a large catalogue of summary genetic association statistics from previously published GWAS (MR-Base; http://www.mrbase.org) [40]. After applying a Bonferroni correction to account for multiple "look ups" of phenotypic traits with all 7 SNPs examined $(p<0.05 / \mathrm{x}$, where $\mathrm{x}$ represents the number of phenotypic trait "look ups" performed; 859 to 1,060 look-ups performed with corresponding corrected $p$ value thresholds: $5.8 \times 10^{-5}$ to $4.7 \times 10^{-5}$ across seven SNPs), we identified two SNPs (rs780094, rs 1550532) that associated with multiple traits in MR-Base. rs780094 was robustly associated $\left(p<4.8 \times 10^{-5}\right)$ with various measures of lipids, insulin, and anthropometric traits and rs 1550532 was robustly associated $\left(p<4.8 \times 10^{-5}\right)$ with inflammatory bowel disease; these traits have all been hypothesized to influence prostate cancer risk [41-44]. Additionally, rs1550532 was strongly associated with levels of multiple "unknown metabolites" from untargeted GWAS of metabolomic studies [45]. Given that these two SNPs could influence prostate cancer risk through biological pathways independent of calcium (i.e. horizontal pleiotropy), we removed them from our genetic instrument. Consequently, our genetic instrument for calcium used five SNPs that we assessed as being exclusively associated with serum calcium (rs17251221, rs10491003, rs7481584, rs7336933, rs1570669).

\section{Statistical analysis}

We generated estimates of the proportion of variance in serum calcium for our genetic instrument $\left(R^{2}\right)$ and F-statistics to examine the strength of our instruments and to test for weak instrument bias (a reduction in statistical power to reject the null hypothesis when an instrument explains only a small proportion of variance in an exposure), using methods previously described [46]. Power calculations were performed using previously reported methods [47] to determine whether we had sufficient sample size to identify effect sizes in our MR analyses that were of a similar magnitude to those reported in the observational literature.

We first examined the effect of serum calcium on overall and advanced prostate cancer for individual SNPs, using the Wald ratio to generate beta-coefficients, and the delta method approximation of the standard error. SNPs were then combined into a multi-allelic genetic instrument (to increase the variance explained in serum calcium) and the causal effect of this instrument on overall and advanced prostate cancer was examined using a maximum likelihood-based approach [48]. For both individual-SNP and multi-allelic instrument analyses, the effect of serum calcium on prostate cancer was scaled to represent a $0.5 \mathrm{mg} / \mathrm{dL}$ increase $(\sim 1 \mathrm{SD}) . I^{2}$ statistics were calculated to determine the percentage of heterogeneity across SNPs in causal estimates due to variability beyond chance and Cochran's $Q$ test was used to test homogeneity across SNPs in causal estimates [49]. Maximum-likelihood estimates were then generated using fixed-effects or random-effects models depending on heterogeneity of causal effect estimates across SNPs in multi-allelic instruments. $p$ values were generated using a $\mathrm{t}$-distribution with $\mathrm{N}-1$ degrees of freedom where $N$ is the number of SNPs utilized in the instrument.

To examine the presence of directional pleiotropy (where the horizontally pleiotropic effect across a genetic instrument do not average to zero) from unmeasured traits, we performed two sensitivity analyses: MR-Egger regression [50] and the weighted median estimator approach [51]. MREgger relaxes the exclusion restriction criterion and thus can provide unbiased estimates of causal effects even when all 
IVs in an instrument are invalid through violation of this assumption. This approach performs a weighted generalized linear regression of the SNP-outcome coefficients on the SNP-exposure coefficients with an unconstrained intercept term. Provided that the InSIDE (Instrument Strength Independent of Direct Effect) assumption is met (that no association exists between the strength of gene-exposure associations and the strength of bias due to horizontal pleiotropy) and that measurement error in the genetic instrument is negligible ("No Measurement Error" or NOME assumption) [52], the slope generated from MR-Egger regression can provide an estimate of the causal effect of calcium on prostate cancer that is adjusted for directional pleiotropy and the intercept term can provide a formal statistical test for directional pleiotropy. To test NOME, we generated weighted $I_{G X}^{2}$ values for overall and advanced prostate cancer analyses to quantify the expected dilution of MREgger estimates due to NOME violations [52]. The weighted median estimator (WME) approach provides an estimate of the weighted median of a distribution in which individual IV causal estimates in an instrument are ordered and weighted by the inverse of their variance. Unlike MR-Egger which can provide an unbiased causal effect even when all IVs are invalid, WME requires that at least $50 \%$ of the information in a multi-allelic instrument is coming from SNPs that are valid IVs in order to provide an unbiased estimate of a causal effect in an MR analysis. However, the WME has two advantages over MR-Egger in that it provides improved precision as compared to the latter and does not rely on the InSIDE assumption.

We also performed a leave-one-out permutation analysis to examine whether any of our results were driven by any individual SNP from our multi-allelic instrument.

All statistical analyses were performed using $\mathrm{R}$ version 3.3.1.

\section{Results}

Our genetic instrument explained $0.71 \%$ of variance in serum calcium levels. The corresponding F-statistic for our instrument (86.2) suggested that our instrument was unlikely to suffer from weak instrument bias [53]. Power calculations suggested that we would have $80 \%$ power to detect an OR of at least 1.25 (or, conversely a protective OR of at least 0.80 ) per $0.5 \mathrm{mg} / \mathrm{dL}$ increase in serum calcium on overall prostate cancer risk at an alpha level (false positive) of 5\%. For advanced prostate cancer, we had $80 \%$ power to detect an OR of at least 1.81 (or a protective OR at least 0.55 ), which would be of similar magnitude to effect estimates reported in the largest observational study of fatal prostate cancer to date (HR [Hazard Ratio] 1.66 per $0.5 \mathrm{mg} / \mathrm{dL}$ increase in serum calcium) [26].

Estimates of causal effects of individual calcium SNPs per $0.5 \mathrm{mg} / \mathrm{dL}$ increase in serum calcium on overall and advanced prostate cancer are presented in Table 1. Individually, there was little evidence that any of the five SNPs were causally associated with overall or advanced prostate cancer.

\section{Overall prostate cancer}

In an MR analysis combining the five serum calcium-related SNPs into a multi-allelic genetic instrument, there was weak evidence of a protective effect of serum calcium on prostate cancer risk (OR per $0.5 \mathrm{mg} / \mathrm{dL}$ increase in calcium: 0.83 , 95\% CI 0.63-1.08; $p=0.12$ ) (Table 2). Effect estimates were similar using the weighted median estimator (OR 0.80, 95\% CI 0.58-1.12) and MR-Egger (OR 0.87, 95\% CI 0.46-1.64). The MR-Egger intercept parameter did not suggest evidence of directional pleiotropy (OR 1.00, $p=0.76$ ).

Table 1 Descriptive statistics of calcium SNPs and estimates of their causal effects on overall and advanced prostate cancer in PRACTICAL

\begin{tabular}{|c|c|c|c|c|c|c|c|c|}
\hline SNP & $\mathrm{Chr}$ & Gene(s) & EA & NEA & $\begin{array}{l}\text { Overall prostate can- } \\
\text { cer OR }(95 \% \mathrm{CI})\end{array}$ & $p$ value & $\begin{array}{l}\text { Advanced prostate } \\
\text { cancer OR }(95 \% \mathrm{CI})\end{array}$ & $p$ value \\
\hline rs 17251221 & 3 & CASR & G & A & $0.84(0.65-1.09)$ & 0.18 & $0.83(0.51-1.35)$ & 0.45 \\
\hline rs10491003 & 10 & GATA3 & $\mathrm{T}$ & $\mathrm{C}$ & $0.56(0.28-1.15)$ & 0.12 & $1.58(0.42-5.93)$ & 0.50 \\
\hline rs7481584 & 11 & $C A R S$ & G & A & $0.72(0.37-1.40)$ & 0.33 & $1.21(0.34-4.23)$ & 0.77 \\
\hline rs7336933 & 13 & DGKH; KIAA0564 & G & A & $1.36(0.68-2.70)$ & 0.39 & $1.60(0.44-5.80)$ & 0.48 \\
\hline rs 1570669 & 20 & $C Y P 24 A 1$ & G & A & $0.66(0.35-1.25)$ & 0.20 & $1.01(0.31-3.29)$ & 0.99 \\
\hline
\end{tabular}

EA reflects the allele that increases serum calcium levels. OR $(95 \% \mathrm{CI})$ represents the exponential increase in odds for each $0.5 \mathrm{mg} / \mathrm{dL}$ increase in serum calcium

Chr chromosome, EA effect allele, NEA non-effect allele, OR odds ratio, 95\% CI 95\% confidence interval 
Table 2 Mendelian randomization derived causal effects of a $0.5 \mathrm{mg} / \mathrm{dL}$ increase in serum calcium on overall and advanced prostate cancer using a multi-allelic instrument in PRACTICAL

\begin{tabular}{lllll}
\hline & $\begin{array}{l}\text { Maximum like- } \\
\text { lihood estimate } \\
\text { OR }(95 \% \mathrm{CI})^{\mathrm{a}}\end{array}$ & $\begin{array}{l}\text { Weighted median estimator } \\
\text { OR }(95 \% \mathrm{CI})\end{array}$ & $\begin{array}{l}\text { MR-Egger regression } \\
\text { OR }(95 \% \mathrm{CI})\end{array}$ & $\begin{array}{l}\text { MR-Egger regres- } \\
\text { sion intercept } \\
\text { term } \\
p \text { value }\end{array}$ \\
\hline $\begin{array}{l}\text { Overall pros- } \\
\text { tate cancer } \\
(n=72,729)^{\mathrm{b}}\end{array}$ & $0.83(0.63-1.08)$ & $0.80(0.58-1.12)$ & $0.87(0.46-1.64)$ & 0.76 \\
$\begin{array}{c}\text { Advanced } \\
\text { prostate } \\
\text { cancer } \\
(n=33,498)^{\mathrm{c}}\end{array}$ & $0.98(0.57-1.70)$ & $0.92(0.50-1.66)$ & $0.72(0.24-2.15)$ & 0.42 \\
\hline
\end{tabular}

${ }^{a}$ Odds ratio [OR] $(95 \%$ confidence interval, CI) represents the exponential increase in odds for each $0.5 \mathrm{mg} / \mathrm{dL}$ increase in serum calcium

${ }^{\mathrm{b}}$ Maximum likelihood estimate obtained using a fixed-effects model $\left(I^{2}=0 \%, \mathrm{Qp}=0.44\right)$

${ }^{\mathrm{c}}$ Fixed-effects model $\left(I^{2}=0 \%, \mathrm{Qp}=0.80\right)$

\section{Advanced prostate cancer}

MR analyses found little evidence for an effect of serum calcium on advanced prostate cancer risk $(0.5 \mathrm{mg} / \mathrm{dL}$ calcium increase: OR 0.98, 95\% CI 0.57-1.70; $p=0.93$ ) (Table 2). Sensitivity analyses to examine directional pleiotropy were consistent with a null effect of serum calcium on advanced prostate cancer.

Calculation of the $I_{G X}^{2}$ statistic suggested little attenuation of our MR-Egger estimates due to measurement error for both overall prostate cancer $\left(I_{G X}^{2}=0.89\right)$ and advanced prostate cancer $\left(I_{G X}^{2}=0.91\right)$, so adjustment of MR-Egger estimates to account for mild dilution bias was not performed [52]. Leave-one-out permutation analyses for overall and advanced prostate cancer did not find evidence that the effect estimate based on the multi-allelic instrument was being driven by any single serum calcium-related SNP (Supplementary Table 1).

\section{Discussion}

Our Mendelian randomization analysis does not support the hypothesis that serum calcium increases the risk of overall or advanced prostate cancer. Indeed, the point estimates were in the opposite direction (though imprecisely estimated) to findings from some observational studies.

Our findings are not consistent with some laboratory studies which have reported a role of calcium in promoting loss of differentiation and increased proliferation of prostate cancer cells [54, 55]. Prospective studies that have examined the association of serum calcium with incident prostate cancer have generated conflicting findings: three did not find strong evidence for an association (HR for upper vs lower tertile: $1.31,95 \%$ CI 0.77-2.20 [23]; OR for upper vs lower quartile: $1.04,95 \%$ CI $0.78-1.39$ [28]; HR per quartile increase: $0.99,95 \%$ CI $0.94-1.03$ [29]), whereas one reported a weak inverse association between calcium and prostate cancer (HR per SD increase: 0.97, 95\% CI 0.85-1.00) [24]. Likewise, some studies that have examined an association between serum calcium and fatal prostate cancer have reported positive risk relationships (HR for upper vs lower tertile: 2.07, 95\% CI 1.06-4.04 [27]; HR for upper vs lower tertile: 2.68 , 95\% CI 1.02-6.99 [23]; HR per $0.1 \mathrm{mmol} / \mathrm{L}$ increase: 1.50 , 95\% CI 1.04-2.17 [26]) whereas others have not found strong evidence of an association (HR per 1-SD increase: 1.00, 95\% CI 0.92-1.09 [24]; HR for upper vs lower quartile: $0.75,95 \%$ CI $0.49-1.15$ [25]). It is plausible that discordance between previously reported observational findings and our MR analysis may reflect residual confounding in the former (e.g. through other dietary, lifestyle, or molecular factors). It is also possible that studies reporting associations of dairy products with subsequent prostate cancer risk may reflect the mediating role of proteins and growth factors (e.g. insulin-like growth factor 1 ) in prostate carcinogenesis, and not calcium per se $[11,56,57]$. Nevertheless, the weak evidence that we found for a potential protective effect of serum calcium on overall prostate cancer is consistent with a meta-analysis of four randomized controlled trials that reported that daily calcium supplementation $(\geq 500 \mathrm{mg} /$ day $)$ reduced prostate cancer risk (RR [Risk Ratio] 0.54, 95\% CI $0.30-0.96, p=0.03)$, though this analysis was only based on 48 men with prostate cancer $(3,297$ and 3,248 in the intervention and control groups, respectively) [58].

Strengths of our analysis include the use of a Mendelian randomization approach to appraise the relationship of serum calcium with prostate cancer risk which should help to minimize or avoid confounding through lifestyle or environmental factors that may have biased findings from previous observational analyses. Further, given the time required for nutritional biomarkers to influence carcinogenesis [59] and the considerable latency period of prostate cancer [60], the use of germline genetic variation as an instrument should allow for sufficient time to confer an effect on 
prostate cancer. This is because MR will estimate the effect of life-long exposure to elevated serum calcium on prostate cancer risk. MR will also offer an additional strength over prospective studies of dietary or serum calcium which can suffer from substantial (albeit, likely non-differential) measurement error: measurement error in genetic studies is often low as modern genotyping technologies provide relatively precise measurement of genetic variants [61]. While residual measurement error in SNP-exposure estimates cannot be ruled out, such non-differential measurement error would likely bias associations toward the null, providing a conservative effect estimate. The use of a twosample MR approach allowed us to utilize summary effect estimates from two large GWAS and thus increase statistical power in our analyses. Though the F-statistic generated for our instrument suggested that weak instrument bias was unlikely, in a two-sample MR setting, weak instrument bias if present would be expected to lead to an attenuated effect estimate. This is in contrast to a one-sample MR analysis in which weak instrument bias will tend to bias effect estimates toward the confounded observational study estimate [36]. Lastly, by obtaining summary effect estimates for both exposure and outcome datasets from GWAS that were restricted to individuals of European descent and adjusted for principal components of ancestry, we reduced (though did not eliminate) the possibility of confounding through population stratification in our MR analyses (though this may limit generalizability of our findings to other ethnicities).

There are limitations to our analysis. First, given the composite characterization of advanced prostate cancer in the summary GWAS data that we obtained (Gleason $\geq 8$, prostate-specific antigen $>100 \mathrm{ng} / \mathrm{mL}$, metastatic disease (M1), or death from prostate cancer), it is difficult to directly compare our findings with those from prospective studies that examined associations between calcium and fatal prostate cancer. Second, though our MR analysis for advanced prostate cancer was sufficiently powered to detect effect sizes compatible with those reported in the observational literature, it was not powered to detect effect sizes of a more modest magnitude. Further identification of independent genetic variants that influence serum calcium (increasing instrument strength further by explaining a larger proportion of the variance in serum calcium) in addition to larger GWAS of advanced prostate cancer will help to improve statistical power for future analyses. A final limitation of our analysis was that we were unable to examine possible non-linear effects of serum calcium on prostate cancer using summarized genetic data, which have been proposed previously [8].

Given that our findings raise the possibility that serum calcium may be protective against prostate cancer, there is a need to follow-up these results in large and independent datasets. Further identification of additional independent genetic variants robustly associated with serum calcium will help to improve precision of future analyses.

In conclusion, our Mendelian randomization analysis does not support the hypothesis that serum calcium increases the risk of overall or advanced prostate cancer.

Acknowledgments The authors' responsibilities were as follows-SJL and CB: conceived the study; JY, KB, RL, CB, and SL: planned the analyses; JY: conducted data analysis; JY, KB, RL: prepared the manuscript; CB, the PRACTICAL consortium, GDS, RMM, SL: critically revised the manuscript; and all authors: read and approved the final manuscript. None of the authors had any conflicts of interest to declare.

Funding This research was funded by a grant awarded to SJL for 3 years to identify modifiable risk factors for prostate cancer, by the World Cancer Research Fund International (Grant Reference Number: 2015/1421). JY and RL are supported by Cancer Research UK (C18281/A19169) programme grant (the Integrative Cancer Epidemiology Programme Cancer Research UK Research PhD studentships (C18281/A20988 to JY and RL). RMM is also supported by the National Institute for Health Research (NIHR) Bristol Biomedical Research Centre. All authors are further supported by a Cancer Research UK (C18281/A19169) programme grant (the Integrative Cancer Epidemiology Programme) and are part of the Medical Research Council Integrative Epidemiology Unit at the University of Bristol supported by the Medical Research Council (MC_UU_12013/1, MC_ UU_12013/2, and MC_UU_12013/3) and the University of Bristol. The funders played no role in the design, implementation, analysis, or interpretation of the data in this study.

Open Access This article is distributed under the terms of the Creative Commons Attribution 4.0 International License (http://creativeco mmons.org/licenses/by/4.0/), which permits unrestricted use, distribution, and reproduction in any medium, provided you give appropriate credit to the original author(s) and the source, provide a link to the Creative Commons license, and indicate if changes were made.

\section{References}

1. Global Burden of Disease Cancer Collaboration (2015) et al The global burden of cancer 2013. JAMA Oncol 1(4):505-527

2. Gann PH (2002) Risk factors for prostate cancer. Rev Urol 4(Suppl 5):S3-S10

3. Center MM et al (2012) International variation in prostate cancer incidence and mortality rates. Eur Urol 61(6):1079-1092

4. Wong MC et al (2016) Global incidence and mortality for prostate cancer: analysis of temporal patterns and trends in 36 countries. Eur Urol 70(5):862-874

5. Haenszel W, Kurihara M (1968) Studies of Japanese migrants. I. Mortality from cancer and other diseases among Japanese in the United States. J Natl Cancer Inst 40(1):43-68

6. McCredie M, Williams S, Coates M (1999) Cancer mortality in East and Southeast Asian migrants to New South Wales, Australia, 1975-1995. Br J Cancer 79(7-8):1277-1282

7. Thomas DB, Karagas MR (1996) Migrant studies. In: Schottenfeld D, Fraumeni JF (eds) Cancer epidemiology and prevention, Oxford University Press, New York, pp 236-254

8. Butler LM et al (2010) Calcium intake increases risk of prostate cancer among Singapore Chinese. Cancer Res 70(12):4941-4948 
9. Gao X, LaValley MP, Tucker KL (2005) Prospective studies of dairy product and calcium intakes and prostate cancer risk: a meta-analysis. J Natl Cancer Inst 97(23):1768-1777

10. Tseng $\mathrm{M}$ et al (2005) Dairy, calcium, and vitamin $\mathrm{D}$ intakes and prostate cancer risk in the National Health and nutrition examination epidemiologic follow-up study cohort. Am J Clin Nutr 81(5):1147-1154

11. Aune D et al (2015) Dairy products, calcium, and prostate cancer risk: a systematic review and meta-analysis of cohort studies. Am J Clin Nutr 101(1):87-117

12. Chan JM et al (1998) Dairy products, calcium, phosphorous, vitamin D, and risk of prostate cancer (Sweden). Cancer Causes Control 9(6):559-566

13. Giovannucci E et al (2006) A prospective study of calcium intake and incident and fatal prostate cancer. Cancer Epidemiol Biomark Prev 15(2):203-210

14. Giovannucci E et al (1998) Calcium and fructose intake in relation to risk of prostate cancer. Cancer Res 58(3):442-447

15. Allen NE et al (2008) Animal foods, protein, calcium and prostate cancer risk: the European Prospective Investigation into Cancer and Nutrition. Br J Cancer 98(9):1574-1581

16. Park SY et al (2007) Calcium, vitamin D, and dairy product intake and prostate cancer risk: the Multiethnic Cohort Study. Am J Epidemiol 166(11):1259-1269

17. Peacock M (2010) Calcium metabolism in health and disease. Clin J Am Soc Nephrol 5(Suppl 1):S23-S30

18. Schwartz GG (2009) Is serum calcium a biomarker of fatal prostate cancer? Future Oncol 5(5):577-580

19. Konety BR et al (1996) The role of vitamin D in normal prostate growth and differentiation. Cell Growth Differ 7(11):1563-1570

20. Schwartz GG et al (1995) 1,25-Dihydroxy-16-ene-23-yne-vitamin D3 and prostate cancer cell proliferation in vivo. Urology 46(3):365-369

21. Schwartz GG et al (1997) 1 alpha,25-Dihydroxyvitamin D (calcitriol) inhibits the invasiveness of human prostate cancer cells. Cancer Epidemiol Biomark Prev 6(9):727-732

22. Giovannucci E (1998) Dietary influences of $1,25(\mathrm{OH}) 2$ vitamin $D$ in relation to prostate cancer: a hypothesis. Cancer Causes Control 9(6):567-582

23. Skinner HG, Schwartz GG (2008) Serum calcium and incident and fatal prostate cancer in the National Health and Nutrition Examination Survey. Cancer Epidemiol Biomark Prev 17(9):2302-2305

24. Van Hemelrijck M et al (2012) Serum calcium and incident and fatal prostate cancer in the Swedish AMORIS study. Cancer Causes Control 23(8):1349-1358

25. Brandstedt J et al, Vitamin D (2016) PTH, and calcium in relation to survival following prostate cancer. Cancer Causes Control 27(5):669-677

26. Schwartz GG, Skinner HG (2012) A prospective study of total and ionized serum calcium and time to fatal prostate cancer. Cancer Epidemiol Biomark Prev 21(10):1768-1773

27. Skinner HG, Schwartz GG (2009) A prospective study of total and ionized serum calcium and fatal prostate cancer. Cancer Epidemiol Biomark Prev 18(2):575-578

28. Brandstedt J et al, Vitamin D (2012) PTH, and calcium and the risk of prostate cancer: a prospective nested case-control study. Cancer Causes Control 23(8):1377-1385

29. Halthur $\mathrm{C}$ et al (2009) Serum calcium and the risk of prostate cancer. Cancer Causes Control 20(7):1205-1214

30. Smith GD, Ebrahim S (2001) Epidemiology-is it time to call it a day? Int J Epidemiol 30(1):1-11

31. Lawlor DA et al (2004) Those confounded vitamins: what can we learn from the differences between observational versus randomised trial evidence? Lancet 363(9422):1724-1727

32. Davey Smith G, Ebrahim S (2003) 'Mendelian randomization': can genetic epidemiology contribute to understanding environmental determinants of disease? Int $\mathrm{J}$ Epidemiol 32(1): $1-22$

33. Yarmolinsky J et al (2018) Causal inference in cancer epidemiology: what is the role of mendelian randomization? Cancer Epidemiol Biomark Prev 27(9):995-1010

34. O'Seaghdha CM et al (2013) Meta-analysis of genome-wide association studies identifies six new Loci for serum calcium concentrations. PLoS Genet 9(9):e1003796

35. Schumacher FR et al (2018) Association analyses of more than 140,000 men identify 63 new prostate cancer susceptibility loci. Nat Genet 50(7):928-936

36. Pierce BL, Burgess S (2013) Efficient design for Mendelian randomization studies: subsample and 2-sample instrumental variable estimators. Am J Epidemiol 178(7):1177-1184

37. Amos CI et al (2017) The OncoArray Consortium: a network for understanding the genetic architecture of common cancers. Cancer Epidemiol Biomark Prev 26(1):126-135

38. 1000 Genomes Project Consortium (2015) et al A global reference for human genetic variation. Nature 526(7571):68-74

39. 1000 Genomes Project Consortium (2010) et al A map of human genome variation from population-scale sequencing. Nature 467(7319):1061-1073

40. Hemani $\mathrm{G}$ et al (2018) The MR-Base platform supports systematic causal inference across the human phenome. eLife 7:e34408

41. Albanes D et al (2009) Serum insulin, glucose, indices of insulin resistance, and risk of prostate cancer. J Natl Cancer Inst 101(18):1272-1279

42. Gong $\mathrm{Z}$ et al (2006) Obesity, diabetes, and risk of prostate cancer: results from the prostate cancer prevention trial. Cancer Epidemiol Biomark Prev 15(10):1977-1983

43. Jess $\mathrm{T}$ et al (2013) Cancer risk in inflammatory bowel disease according to patient phenotype and treatment: a Danish population-based cohort study. Am J Gastroenterol 108(12):1869-1876

44. Kok DE et al (2011) Blood lipid levels and prostate cancer risk; a cohort study. Prostate Cancer Prostatic Dis 14(4):340-345

45. Shin SY et al (2014) An atlas of genetic influences on human blood metabolites. Nat Genet 46(6):543-550

46. Burgess S, Thompson SG, Collaboration CCG (2011) Avoiding bias from weak instruments in Mendelian randomization studies. Int J Epidemiol 40(3):755-764

47. Burgess S (2014) Sample size and power calculations in Mendelian randomization with a single instrumental variable and a binary outcome. Int J Epidemiol 43(3):922-929

48. Burgess S et al (2015) Using published data in Mendelian randomization: a blueprint for efficient identification of causal risk factors. Eur J Epidemiol 30(7):543-552

49. Higgins JP et al (2003) Measuring inconsistency in meta-analyses. BMJ 327(7414):557-560

50. Bowden J, Smith GD, Burgess S (2015) Mendelian randomization with invalid instruments: effect estimation and bias detection through Egger regression. Int J Epidemiol 44(2):512-525

51. Bowden $\mathrm{J}$ et al (2016) Consistent estimation in mendelian randomization with some invalid instruments using a weighted median estimator. Genet Epidemiol 40(4):304-314

52. Bowden $\mathrm{J}$ et al (2016) Assessing the suitability of summary data for two-sample Mendelian randomization analyses using MR-Egger regression: the role of the I2 statistic. Int J Epidemiol 45(6): 1961-1974

53. Pierce BL, Ahsan H, Vanderweele TJ (2011) Power and instrument strength requirements for Mendelian randomization studies using multiple genetic variants. Int J Epidemiol 40(3):740-752

54. Liao J et al (2006) Extracellular calcium as a candidate mediator of prostate cancer skeletal metastasis. Cancer Res 66(18):9065-9073

55. Ritchie CK et al (1997) Effects of the calciotrophic peptides calcitonin and parathyroid hormone on prostate cancer growth and chemotaxis. Prostate 30(3):183-187 
56. Harrison $\mathrm{S}$ et al (2017) Does milk intake promote prostate cancer initiation or progression via effects on insulin-like growth factors (IGFs)? A systematic review and meta-analysis. Cancer Causes Control 28(6):497-528

57. Roddam AW et al (2008) Insulin-like growth factors, their binding proteins, and prostate cancer risk: analysis of individual patient data from 12 prospective studies. Ann Intern Med 149(7):461-471

58. Bristow SM et al (2013) Calcium supplements and cancer risk: a meta-analysis of randomised controlled trials. Br J Nutr 110(8):1384-1393
59. Sanson-Fisher RW et al (2007) Limitations of the randomized controlled trial in evaluating population-based health interventions. Am J Prev Med 33(2):155-161

60. Etzioni R et al (1998) Asymptomatic incidence and duration of prostate cancer. Am J Epidemiol 148(8):775-785

61. Schatzkin A et al (2009) Mendelian randomization: how it canand cannot-help confirm causal relations between nutrition and cancer. Cancer Prev Res 2(2):104-113 